\title{
Large-eddy simulations of co-annular turbulent jet using a Voronoi-based mesh generation framework
}

\author{
Guillaume A. Brès, Sanjeeb T. Boseł Michael Emorył Frank E. Ham \\ Cascade Technologies Inc., Palo Alto, CA 94303, USA \\ and \\ Oliver T. Schmidt, Georgios Rigas \& Tim Colonius\| \\ California Institute of Technology, Pasadena, CA 91125, USA
}

\begin{abstract}
Large eddy simulations are performed for a cold ideally-expanded dual-stream jet issued from cylindrical co-axial nozzles, with supersonic primary stream (Mach number $M_{1}=1.55$ ) and subsonic secondary stream $\left(M_{2}=0.9\right)$. The geometry includes the internal screw holes used to fasten the two nozzles together and to the plenum chamber. These slanted cylindrical holes over which the secondary stream flows were not covered in the experiment and were seamlessly captured in the computational mesh thanks to a novel grid generation paradigm based on the computation of Voronoi diagrams. A simulation with the screw holes covered is also performed and the preliminary results tends to indicate that these features have minimal impact on the flow and acoustic fields for the present operating conditions. As expected, the present dual-stream configuration with subsonic annular stream surrounding the primary supersonic stream features a reduced shear-layer growth, a longer potential core and a lack of strong Mach wave radiation. A long LES database is currently being collected for analysis and modeling of wavepackets and noise sources in such complex turbulent jets.
\end{abstract}

\section{Nomenclature}

\begin{tabular}{llll}
$c$ & Speed of sound & $x, y, z$ & Cartesian coordinates \\
$D$ & Nozzle exit diameter & $\Delta t$ & Sampling period \\
$d t$ & Time step & $\mu$ & Dynamic viscosity \\
$f$ & Frequency & $\phi$ & Jet inlet angle \\
$M$ & Mach number & $\rho$ & Density \\
$M_{\infty}$ & Wind-tunnel Mach number & $\theta$ & Azimuthal angle \\
$N P R$ & Nozzle pressure ratio & Subscript & \\
$N T R$ & Nozzle temperature ratio & $\infty$ & Free-stream property \\
$p$ & Pressure & $t$ & Total (stagnation) property \\
$R e$ & Reynolds number & 1 & Primary stream property \\
$S t$ & Strouhal number $f D_{1} / U_{1}$ & 2 & Secondary stream property \\
$T$ & Temperature & Superscript \\
$t$ & Time & $*$ & Dimensional quantity \\
$t_{\text {sim }}$ & Total simulation time & \multicolumn{3}{|}{} \\
$U$ & Mean streamwise jet velocity & \multicolumn{3}{|}{}
\end{tabular}

\footnotetext{
* Director of Operations; Senior Research Scientist - Aeroacoustics

${ }^{\dagger}$ Chief Technology Officer

$\ddagger$ Research Scientist

$\S$ President \& CEO

9 Postdoctoral Fellow

" Professor, Dept. of Mechanical Engineering, AIAA Associate Fellow
} 


\section{Introduction}

Over the past several decades, the exponential growth in computing capacity leveraged by scientific simulation software has resulted in unique insights into multi-scale, multi-physics flow phenomena, including jet aeroacoustics. ${ }^{1,2}$ This learning has principally been done in relatively simple, canonical nozzle geometries where careful attention is paid to ensure adequate spatio-temporal resolution is provided and experimental data is available for validation. In contract, the impact has been limited in more complex geometries relevant for engineering applications. The need to generate large, high-quality meshes typically limits the overall time to solution and often has undesirable effects on the solution accuracy. Indeed, in the NASA's CFD Vision 2030 report, ${ }^{3}$ mesh generation is highlighted as one of the critical bottleneck in the CFD workflow. At the same time, it is quite likely that meshes are here to stay, as the partition of space that they provide is the foundation for most numerical methods.

Historically, there have been four widely used approaches to addressing the mesh-generation challenge in computational fluid dynamics: Cartesian (cut-cell, immersed boundary), multi-block structured meshes, overset grids and generalized unstructured grids (e.g., hexahedral/tetrahedral/prismatic). The latter method with hexahedral-dominant grids and adaptive mesh refinement has been successfully used for jet noise predictions in relatively simple geometrical configurations. ${ }^{4-6}$ These traditional approaches to mesh generation have centered around explicit construction of cell connectivities and topologies that optimize given mesh quality metrics.

In contrast, a novel mesh generation paradigm based on the computation of Voronoi diagrams has been recently developed at Cascade Technologies. Given a set of points where the solution is to be sampled and a description of boundary surfaces, the Voronoi diagram divides the volume based on Euclidean distance and uniquely defines a mesh. By construction, the resulting mesh possesses some desirable properties including orthogonality of face normal and cell displacement vectors. For instance, orthogonal hexahedral meshes are, in many cases, Voronoi diagrams associated with a structured array of points. Coupled with recent developments that allows for the placement of regular self-similar polyhedra (i.e., hexagonally close-packed elements) away from boundaries and the robust calculation of non-convex polyhedra in the vicinity of complex boundary surfaces, this platform allows for the generation of high-quality, body-fitted, conformal meshes suitable for low-dissipation numerical methods necessary for high-fidelity large eddy simulations (LES) of multi-scale turbulent flows.

An overview of the mesh generation approach based on Voronoi diagrams is presented in section II. As an example of application to complex geometries, simulations are performed for supersonic/subsonic dualstream jets issued from co-axial nozzles, including the internal screw holes used to fasten the nozzles to the plenum chamber and between the different sections. As part of a research collaboration with Caltech, a long LES database is being generated to enable advanced analysis towards improvement of the understanding and modeling of the flow physics in turbulent jets. The geometry, experimental configuration, numerical setup and details of the LES database are discussed in section III, with preliminary LES results reported in section IV.

\section{Voronoi diagrams and meshing}

\section{A. Voronoi mesh generation}

The Voronoi-based meshing technology developed at Cascade computes the mesh as the clipped 3D Voronoi diagram of the specified surface geometry and a set of points (i.e., generating points) where the solution is to be sampled. A Voronoi diagram uniquely defines a partition of space based on Euclidean distance from the generating points. An example of a Voronoi diagram is provided in figure 1.

A powerful aspect of using Voronoi diagrams to produce computational meshes is that the definition of the Voronoi vertex locations uniquely describes the Voronoi mesh, including all geometric information (e.g. volumes, face normals and areas), and connectivity (e.g. nearest neighbors). This deterministic connection between the point locations and the resulting mesh dramatically simplifies mesh adaptation, mesh manipulation, and mesh motion. For example, increasing the point density in a particular region corresponds simply to increasing the number of points in that region, or smoothing the mesh corresponds simply to smoothing the distribution of points, free from the troubles of its impact on mesh connectivity.

The generation of a three-dimensional Voronoi mesh inside a complex fluid volume is essentially a threestep iterative but highly-local computation: 


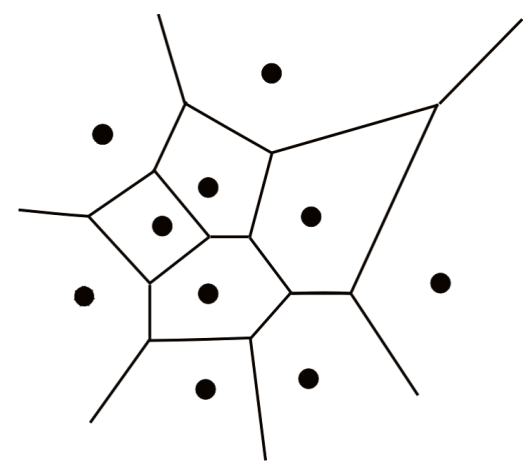

(a) 2D Voronoi diagram

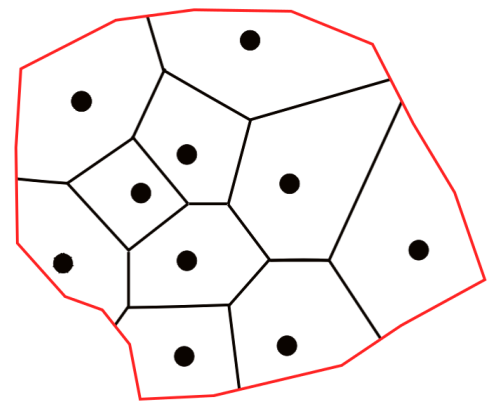

(b) 2D clipped Voronoi diagram

Figure 1. An arbitrary set of generating points (black circles) and the associated 2D Voronoi diagram (black lines). On the right a boundary surface (red lines) confines the mesh to a specific volume; note that the internal cell topology is unchanged.

1. Define the generating point set i.e., the coordinates of the Voronoi vertices that will eventually correspond to the locations of unknowns.

2. Generate the Voronoi diagram, in parallel, from the generating point set including potentially nonconvex boundaries.

3. Potentially smooth or refine the mesh by changing the locations of some or all points, and repeat step 2 .

Another interesting property is that the point cloud and surface are independent objects, i.e., the mesh topology is decoupled from the surface description. The same surface geometry, regardless of tessellation, can be reused during mesh design iterations. Additionally, since the Voronoi diagram is generated solely from point clouds, surfaces can be arbitrarily moved or deformed without folding cell challenges. The Voronoi diagram possesses other desirable properties by construction, such as orthogonality of face normal and cell displacement vectors, enabling computational efficiencies for both the mesh generator and fluid solver.

\section{B. Point clouds as cell centroids}

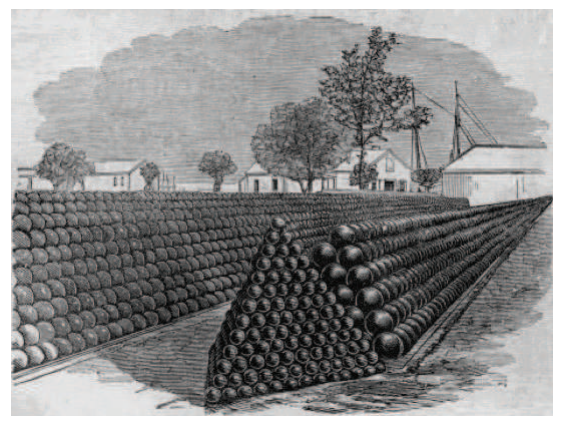

(a) Example of sphere-packing

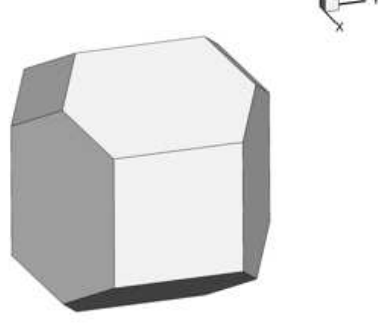

(b) polyhedra cell (front view)

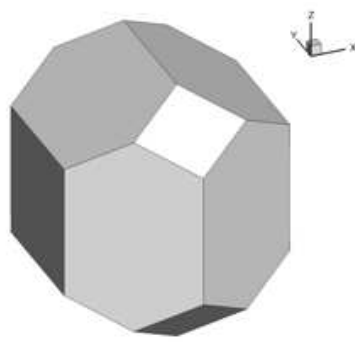

(c) polyhedra cell (side view)

Figure 2. Hexagonal close-packed point distributions corresponding to close-packing of equal spheres (source: Wikipedia) and resulting 14-sided polyhedra cell.

The generation of a Voronoi diagram from a point set is normally performed by either computing the Delaunay triangulation and then taking its dual, or by directly computing the Voronoi diagram from its geometric definition. ${ }^{7}$ As the generation of the Delaunay triangulation in $3 \mathrm{D}$ has problems associated with degenerate point sets, a parallel method has been developed, based on directly cutting the Voronoi diagram generated primarily from a hexagonal close-packed (HCP) point distributions. Hexagonal close-packing 
refers to the spatial arrangement of points corresponding to stable sphere packing (see figure $2(a)$ ). In this arrangement, points are placed inside the fluid volume of interest using an HCP lattice as a guide when seeding points in the domain. The HCP point distributions uniformly sample volume in a way that is different and, in some metrics, more efficient than Cartesian arrays of points. The polyhedral cells resulting from forming the Voronoi diagram with HCP arrays of points are uniform 14-sided polyhedra, as shown in figure $2(b) \&(c)$.

\section{Mesh smoothing and boundary alignment using Lloyd iteration}

Another compelling reason for using Voronoi diagrams as the mesh for the present simulations is the ability to smooth and iteratively improve the mesh in a robust and stable way. For most traditional meshes, particularly unstructured meshes, smoothing can be a complicated process with few guarantees. Meshes can fold, and modifications to the connectivity are tedious. For Voronoi diagrams, however, the connectivity is directly and uniquely determined from the point locations, so smoothing algorithms need only consider the smoothing of the point locations.

One such smoothing algorithm is called Lloyd iteration. ${ }^{8}$ Lloyd iteration makes use of the fact that the Voronoi points and the centroids of the resulting Voronoi cells do not necessarily coincide: when they do, the Voronoi mesh is called centroidal. In the most basic implementation of Lloyd iteration, the generating points are moved to the centroids of their Voronoi cells, and the Voronoi mesh is recomputed (see 3). An important property of Lloyd iteration is that it can be used to produce boundary alignment of the generating points near surfaces. It can also be restricted to a subset of the point cloud so that it does not disturb pre-existing point spacing.

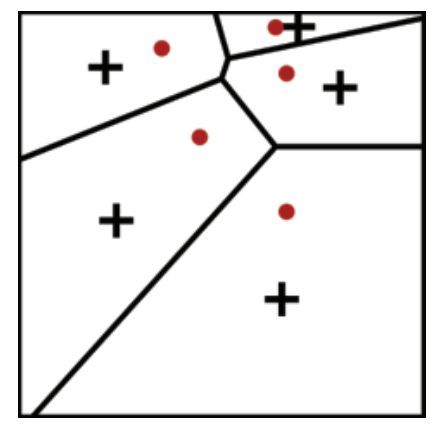

(a) Seed locations

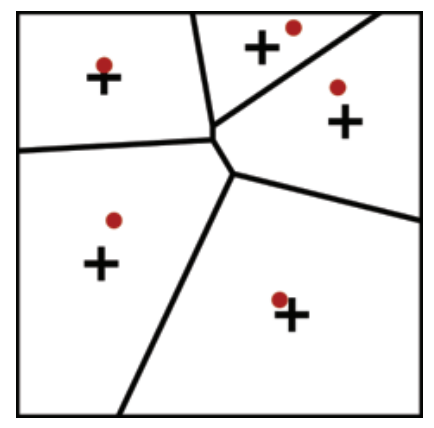

(b) First iteration

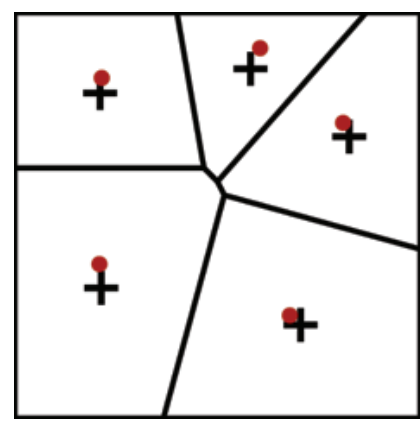

(c) Second iteration

Figure 3. Illustration of 2 Lloyd iterations, where the generating points (red) are moved to the Voronoi cell centroids (black + ) and the Voronoi diagram is recomputed, leading to a more uniform and centroidal mesh (source: Wikipedia).

\section{Application to a co-axial nozzle configuration}

\section{A. Experimental setup}

The present configuration corresponds to a dual-stream jet issued from a cylindrical co-axial nozzle. The primary nozzle diameter is $D_{1}=2$ inches and the combined nozzle diameter is $D_{2}=1.33 D_{1}$. Experiments were conducted at the Georgia Institute of Technology for ideally-expanded cold jet (i.e., acquisition at room temperature), with supersonic primary stream and subsonic secondary stream. While the nozzle design Mach number is $M_{D}=1.6$. it was found that the minimum noise condition is at $M_{1}=1.55$. The secondary nozzle is run at $M_{2}=0.9$ and the bypass ratio is 0.7 based upon the exit areas. For these operating conditions, the experimental measurements include a far-field polar microphone arrays at a constant 12 feet from the nozzle exit, and a linear near-field microphone array at a sideline distance of 24 inches from the nozzle centerline axis. For both arrays, microphones are located at jet inlet angle $\phi=60^{\circ}$ to $150^{\circ}$ in $10^{\circ}$ increment, where $\phi$ is measured from the nozzle centerline upstream axis.

Figure 4 shows the details of the primary and secondary nozzle geometries, including the internal screw holes used to fasten the nozzles to the plenum chamber and between the different sections. These slanted cylindrical holes over which the secondary stream flows were not covered or filled other than by the screws 
in the experiment and are therefore included in the computational domain for the configuration labelled "SH". While such features would have been particularly challenging to mesh with traditional approaches (or even with the previous unstructured grid adaptation framework), the computation of Voronoi diagram near/within the holes does not present any particular difficulty and, much like in any other part of the volume, the resolution in these regions can be controlled by the generating points cloud density. To illustrate this concept, figure 5 shows the surface mesh and a planar cut of the volume mesh for a very coarse grid with refinement near the screw holes. A second configuration labelled "noSH" is also considered, where the nozzle surface geometries were modified with a CAD package to seamlessly cover the screw holes.

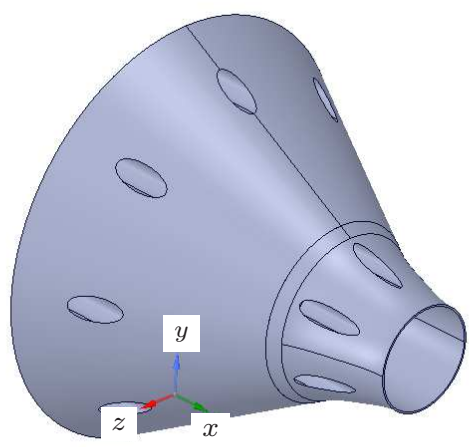

(a) Primary nozzle

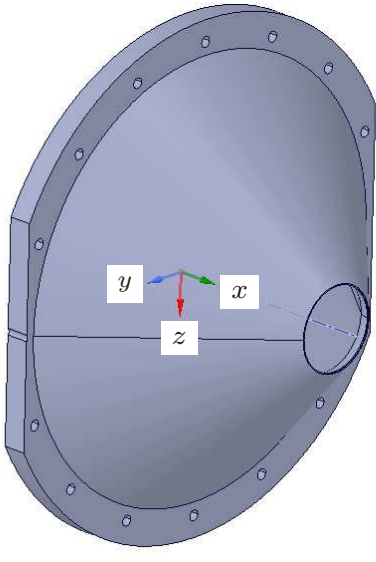

(b) Secondary nozzle

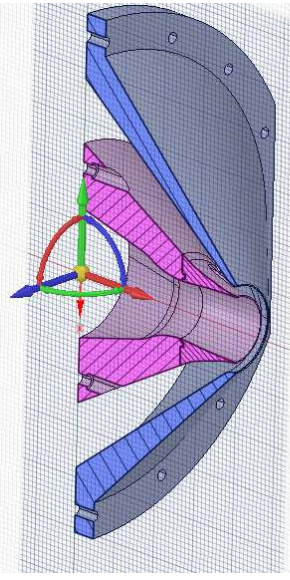

(c) Full configuration

Figure 4. Details of the cylindrical co-axial nozzle geometry

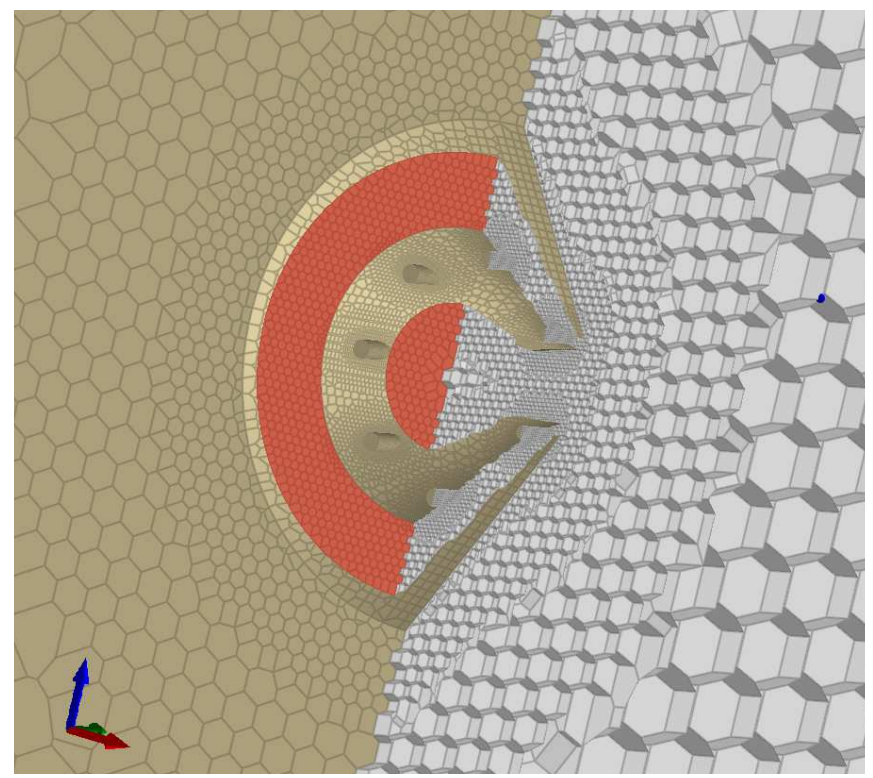

Figure 5. Example of coarse Voronoi mesh of the co-axial nozzle, including the internal screw holes

\section{B. Numerical setup}

For the present work, the simulations and analysis are performed solely within the Voronoi framework, with emphasis on near-wall mesh refinement and modeling inside the nozzle, based on previous LES studies of turbulent jets. ${ }^{9}$ Aside from the differences in mesh topology and corresponding numerics, the setup and methodologies used for the present work are mostly unchanged (see Ref. 6 for further details) and are only briefly summarized here. 
The LES data uses the following nondimensionalization:

$$
\rho=\frac{\rho^{*}}{\rho_{\infty}^{*}}, \quad p=\frac{p^{*}}{\rho_{\infty}^{*} c_{\infty}^{*}{ }^{2}}, \quad T=\frac{T^{*}}{T_{\infty}^{*}}, \quad u=\frac{u^{*}}{c_{\infty}^{*}}, \quad x=\frac{x^{*}}{D^{*}}, \quad t=\frac{t^{*} c_{\infty}^{*}}{D^{*}}, \quad f=\frac{f^{*} D^{*}}{c_{\infty}^{*}},
$$

where the superscript $*$ refers to the dimensional quantity, and the subscript $\infty$ denotes the ambient (freestream) property. The nondimensionalization is based on the primary nozzle diameter $D=D_{1}$ and the ambient speed of sound $c_{\infty}=\sqrt{\gamma p_{\infty} / \rho_{\infty}}$, where $\gamma=1.4$. The resulting form of the ideal gas law is $p=\rho T / \gamma$.

The operating conditions are summarized in table 1 . The jet and acoustic Mach numbers are $M_{1(2)}=$ $U_{1(2)} / c_{1(2)}$ and $M a_{1(2)}=U_{1(2)} / c_{\infty}$ respectively, where the subscript 1 is for the primary jet and 2 for the secondary jet, and $U$ is the mean (time-averaged) streamwise jet velocity. The nozzle pressure ratio and nozzle temperature ratio are defined as $N P R=P_{t} / P_{\infty}$ and $N T R=T_{t} / T_{\infty}$, respectively, and match the experimental conditions. Here, the subscript $t$ refers to the stagnation (total) property. For both experiment and simulation, the Reynolds number based on the primary jet is $R e=\rho_{1} U_{1} D / \mu_{1} \approx 2.9 \times 10^{6}$.

\begin{tabular}{|c|c|c|c|c|c|c|c|c|c|c|}
\hline$N P R_{1}$ & $N P R_{2}$ & $N T R_{1}$ & $N T R_{2}$ & $M_{1}$ & $M_{2}$ & $M a_{1}$ & $M a_{2}$ & $T_{1} / T_{\infty}$ & $T_{2} / T_{\infty}$ & $R e$ \\
\hline \hline 3.93 & 1.69 & 0.99 & 0.99 & 1.55 & 0.9 & 1.26 & 0.83 & 0.67 & 0.85 & $2.9 \times 10^{6}$ \\
\hline
\end{tabular}

Table 1. Summary of operating conditions.

The nozzle geometry (with primary exit centered at $(0,0,0)$ ) is explicitly included in the axisymmetric computational domain, which extends from approximately $-3.5 D$ to $80 D$ in the streamwise $(x)$ direction and flares in the radial direction from $20 D$ to $40 D$. For the present dual-stream configuration, a slower growth of the shear-layer and longer potential core are expected because the secondary jet reduces the shear between the supersonic jet and stationary ambient fluid, leading to weaker instability waves and less (or no) mach wave radiation. ${ }^{10}$ Therefore, the domain (and the main grid refinement region, see next section) has been extended in the streamwise direction, compared to the typical numerical setup for single stream jets.

A very slow coflow at Mach number $M_{\infty}=0.009$ is imposed outside the nozzle in the simulation $\left(M_{\infty}=0\right.$ in the experiment), to prevent any spurious recirculation and facilitate flow entrainment. Sponge layers and damping functions are applied to avoid spurious reflections at the boundary of the computational domain. ${ }^{11,12}$ The Vreman ${ }^{13}$ sub-grid model is used to account for the physical effects of the unresolved turbulence on the resolved flow. To reduce near wall grid resolution requirements, wall modeling is applied on both nozzle interior surfaces. For the wall-modeled boundaries, the LES uses a novel implementation of the wall-stress modeling approach based on equilibrium boundary layer assumptions. ${ }^{14,15}$ All the other solid surfaces are treated as no-slip adiabatic wall.

Far-field noise predictions are performed using an efficient massively-parallel implementation ${ }^{6}$ of the frequency-domain permeable formulation ${ }^{16}$ of the Ffowcs Williams \& Hawkings ${ }^{17}$ (FW-H) equation. The FW-H data is recorded on three conical surfaces extending from the nozzle exit at $r=1 D$ to $x=40 D$, with a slope of $0.04,0.055$ and 0.07 respectively. Again, the parameters of the FW-H surfaces have been modified to take into consideration the longer potential core and lower jet spreading rate for the dual-stream configuration. For the treatment of the FW-H outflow disk, the method of "end-caps" of Shur et al. ${ }^{18}$ is applied for $x>35 D$, where the complex far-field pressure predicted from eleven FW-H surfaces with the same shape but outflow disks at different streamwise locations are phase-averaged.

\section{Voronoi mesh}

To properly capture the development of the internal boundary layers, past jet studies ${ }^{9}$ have suggested that near-wall mesh refinement is needed inside the nozzle, in combination with wall modeling. For the previous unstructured grid adaptation framework, the process requires the generation of a hexahedral-dominant coarse grid as starting point and the specification of target length scales throughout the domain: the mesh adaptation tool then refines elements locally to match the target length scale. For the novel Voronoi mesh framework, there is no initial coarse grid needed (which can potentially be a major bottleneck for realistic complex geometries), the refined mesh is directly computed from the surface description and the specification of the set of generating points where the point density can precisely control the target length scales. As 
the polyhedral cells resulting from the generation of the Voronoi diagram with HCP point distributions are essentially isotropic, the representative length scale for the cell size is $\Delta=v^{1 / 3}$.

The same grid resolution strategy is applied to both configurations with and without screw holes. Near the internal walls and nozzle lips up to $x=1 D$, the target length scale is set to $\Delta=0.005 D$. The length scale is then doubled in two nested refinement windows along both liplines up to $x=15 D$. Finally, the jet plume is fully enclosed in a conical refinement window with $\Delta=0.04 D$ up to $x=35 D$. The rest of the domain is filled with successively coarser mesh, up to the boundaries. Figure 6 shows a planar cut of the Voronoi mesh for the configuration SH50M with screw holes.

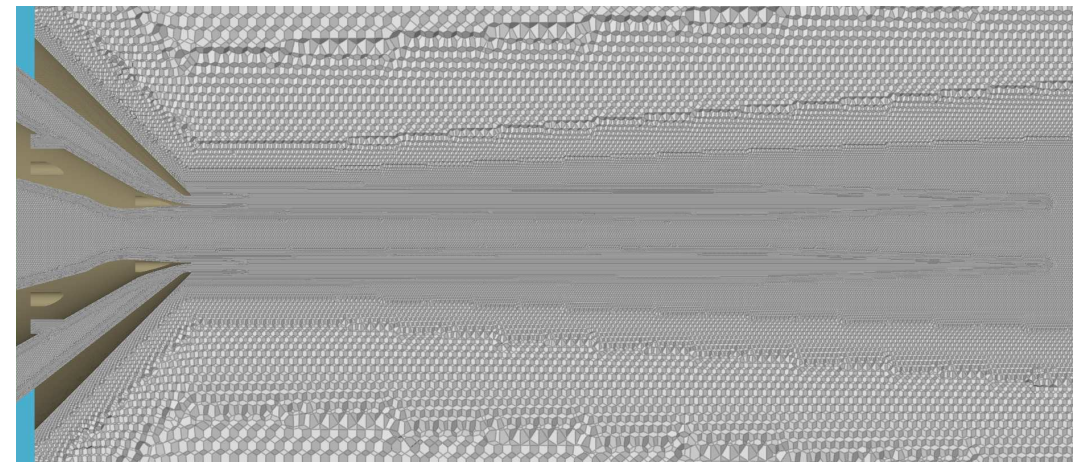

(a) Overview in mid-section plane $z=0$

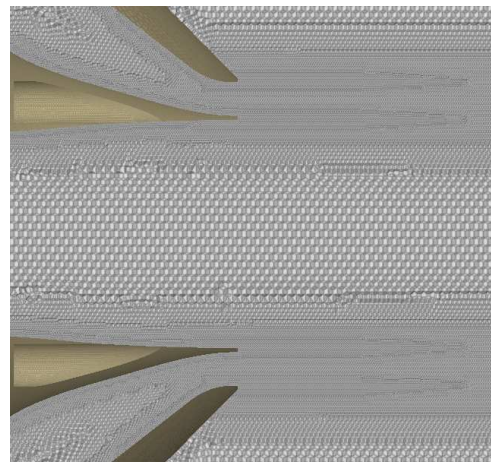

(b) zoomed-in view at $z=0$

Figure 6. Voronoi mesh visualization for the configuration SH50M with screw holes. Only part of the computational domain is shown.

\section{LES database}

Similarly to previous jet database generation, ${ }^{5}$ the LES data in the jet plume is interpolated from the original unstructured LES grid onto a structured cylindrical grid to facilitate post-processing and analysis. This grid is designed such that the resolution approximately corresponds to the underlying LES resolution and limits extrapolation. The three-dimensional cylindrical grid extents to $0 \leq x / D \leq 40$ and $0 \leq r / D \leq 6$, with $\left(n_{x}, n_{r}, n_{\theta}\right)=(800,128,128)$, where $n_{x}, n_{r}$ and $n_{\theta}$ are the number of points in the streamwise, radial and azimuthal direction, respectively. The points are equally-spaced in the azimuthal direction to enable simple azimuthal decomposition in Fourier space. The grid spacing for the structured cylindrical grid is shown figure 7. The point positions $x, y, z$ and the instantaneous value of the primitives variables density $\rho$, velocity (in cartesian coordinate) $u, v, w$ and pressure $p-1 / \gamma$ are saved as single-precision ("float") numbers to reduce disk space.

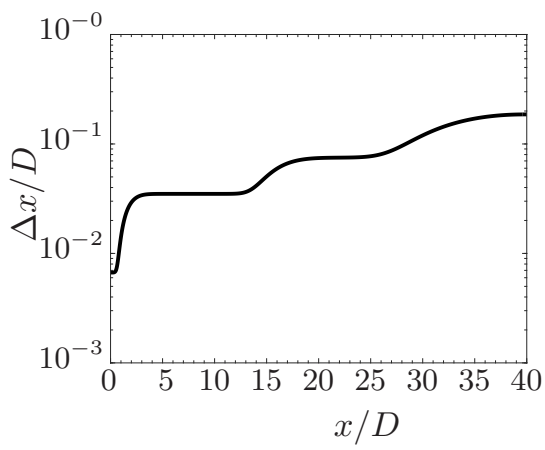

(a) streamwise resolution

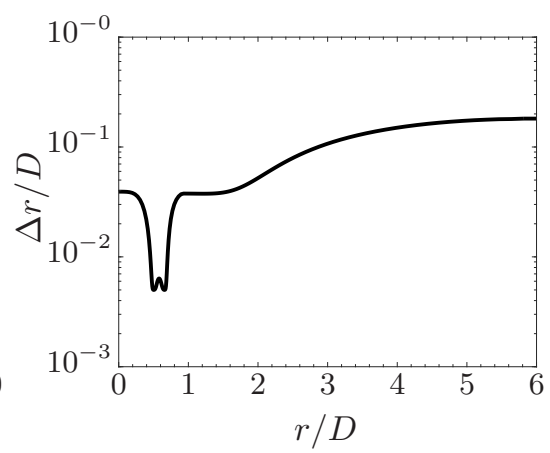

(b) radial resolution

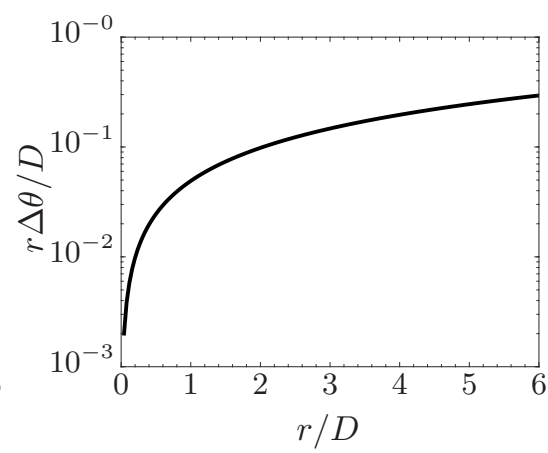

(c) azimuthal resolution

Figure 7. Representation (in logarithmic scale) of the mesh resolution in the jet plume for the structured cylindrical grid used for the LES database. 


\section{Preliminary results}

At this stage, the statistics and LES database have been collected for only 200 acoustics time units, more complete analyses and comparisons with the experimental measurements will be presented in future publication. Table 2 lists the simulation parameters and settings for the LES cases with and without the screw holes, including the time step $d t$, the total simulation time $t_{\text {sim }}$ for the collection of statistics and data (after the initial transient is removed), and the sampling period $\Delta t$ for the recording for the LES database. For both cases, the sampling period for the recording of the FW-H surface data is $\Delta t_{F W H} c_{\infty} / D=0.05$.

\begin{tabular}{|c|c|c|c|c|c|c|}
\hline Case name & Screw holes & Mesh size & Wall model & $d t c_{\infty} / D$ & $\Delta t c_{\infty} / D$ & $t_{s i m} c_{\infty} / D$ \\
\hline \hline noSH50M_WM & no & $50.7 \times 10^{6}$ & $\times$ & 0.001 & 0.1 & 200 \\
SH50M_WM & yes & $50.8 \times 10^{6}$ & $\times$ & 0.001 & - & 200 \\
\hline
\end{tabular}

Table 2. Simulation parameters of the LES cases

\section{A. Flow field visualization and statistics}

Figure 8 shows the instantaneous temperature and pressure fields in the mid-section plane for the noSH50M_WM configuration without screw holes, as an overview visualization of both internal and external flow field, for $-3.5 \leq x / D \leq 26.5$. Zoom-in views of the instantaneous temperature and velocity fields near the nozzle exit are presented in figure 10, for different planes cutting through the screw holes for both SH50M_WM and noSH50M_WM cases. In these figures, the contour ranges (black to white) are $0.5 \leq T / T_{\infty} \leq 1,-0.01 \leq$ $p / p_{\infty}-1 \leq 0.01$ and $0.1 \leq\left|u / c_{\infty}\right| \leq 1.3$. Statistics of the streamwise velocity (non-dimensionalized by the ambient speed of sound) are shown in figure 9 for the whole jet plume in the noSH50M_WM configuration, and in figure 11 near the nozzle exit in both LES cases.
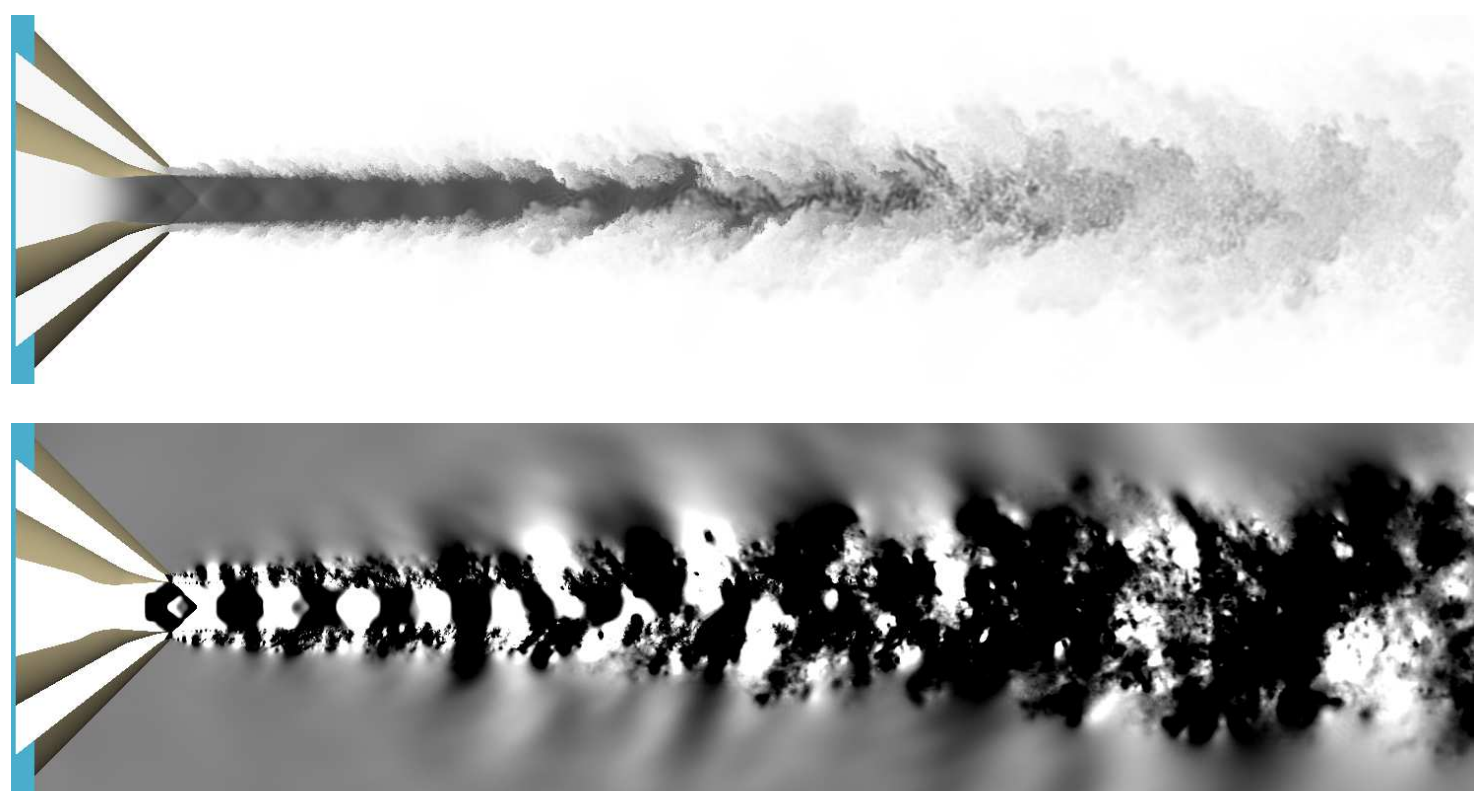

Figure 8. Instantaneous temperature (top) and pressure field (bottom) in mid-section plane for the configuration without screw holes (case noSH5OM_WM).

Overall, the thin (nominally-turbulent) nozzle boundary layers issued from both nozzles immediately transition to turbulent shear layers between the primary and secondary streams, and between the secondary stream and ambient fluid. Similar to laboratory experiments, exact pressure matched condition are challenging to achieve in simulation, and a few residual shock cells are visible near the primary nozzle exit in these figures. As mentioned in section IIIA, the shocks are minimized in the experiment by varying the operating conditions over a small range centered at the design value and determining when the minimum sound level 
is observed. Here, the LES directly match the reported experimental conditions and there were no attempts to minimize the shocks in the simulations. As expected for this dual-stream configuration with a subsonic annular stream surrounding the primary supersonic stream, the shear-layer growth is reduced, the potential core is longer and there is no visible Mach wave radiation.
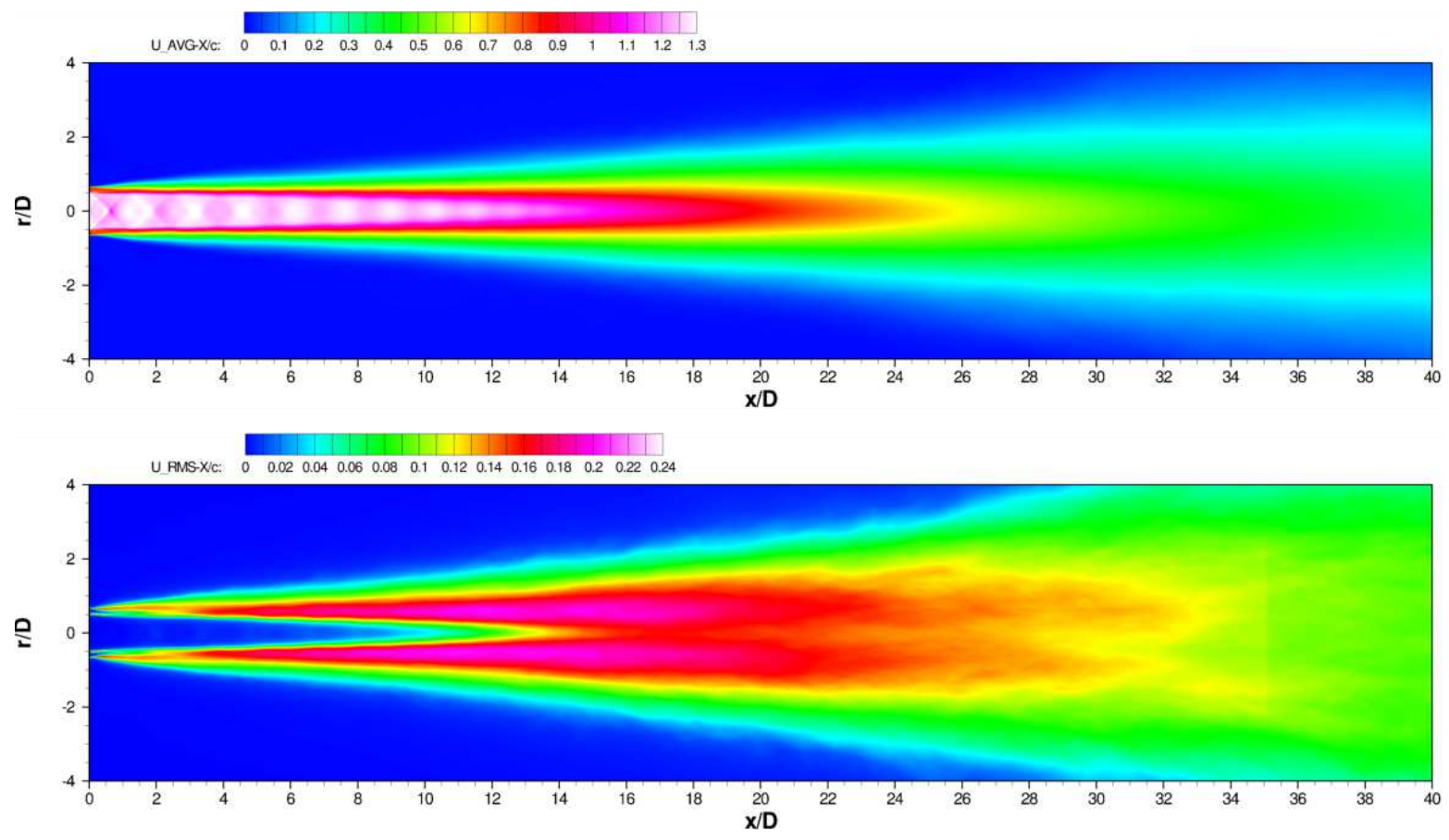

Figure 9. Overview of the time-averaged (top) and RMS (bottom) streamwise velocity in the mid-section plane for the configuration without screw holes (case noSH50M_WM).

As shown in figure 10, inspection of the unsteady flow field inside the nozzle and near the exit shows little differences between the simulations with and without the nozzle internal screw holes. The acoustic Mach number near the larger upstream holes is less than 0.1 , and less than 0.3 near the smaller downstream holes. As the secondary stream is also subject to a strong favorable pressure gradient, these internal features do not seem to produce any meaningful perturbations that could affect the nozzle-exit boundary layer state and initial jet development.

This observation is further confirmed in the time-averaged and RMS streamwise velocity contours presented in figure 11. The results for both simulations SH50M_WM and noSH50M_WM with and without the internal screw holes look qualitatively identical, with the small visible differences likely due to the short simulation time and limited statistical convergence. One interesting feature present in all the locations in figures 10 and 11 is a low speed region with $M a \leq 0.3$ along the primary lipline up $x \approx 0.1 D$, before the shear layers from the two streams start to merge. The rapid collapse of the potential core for the secondary stream results in a relatively thick merged shear layer, as early as 1D downstream of the nozzle exit.

\section{B. Noise predictions}

To investigate the azimuthal symmetry (or lack of) in the radiated noise for the cases with and without screw holes, 24 equally-spaced microphones are distributed along the azimuthal angle, for each inlet angle $\phi$, for both near-field and far-field experimental arrays. For the preliminary near-field results presented in figure 12, the FW-H predictions are performed for all 24 microphones, and the resulting spectra are ensemble-averaged. Additional analysis and comparison with experiment is under way. The PSD is reported in $\mathrm{dB} / \mathrm{St}$, versus frequency in Strouhal $S t=f D / U_{1}$, with respect to the dimensional reference pressure $P_{r e f}^{*}=20 \mu P a$. To compensate for the relatively short time signal in simulation, bin-averaged PSD is computed, with bin size $\Delta S t=0.05$.

Overall, the noise predictions for both configuration are nearly identical, with the main differences in the low frequencies likely due to the limited FW-H data collection. The computations are currently ongoing to 

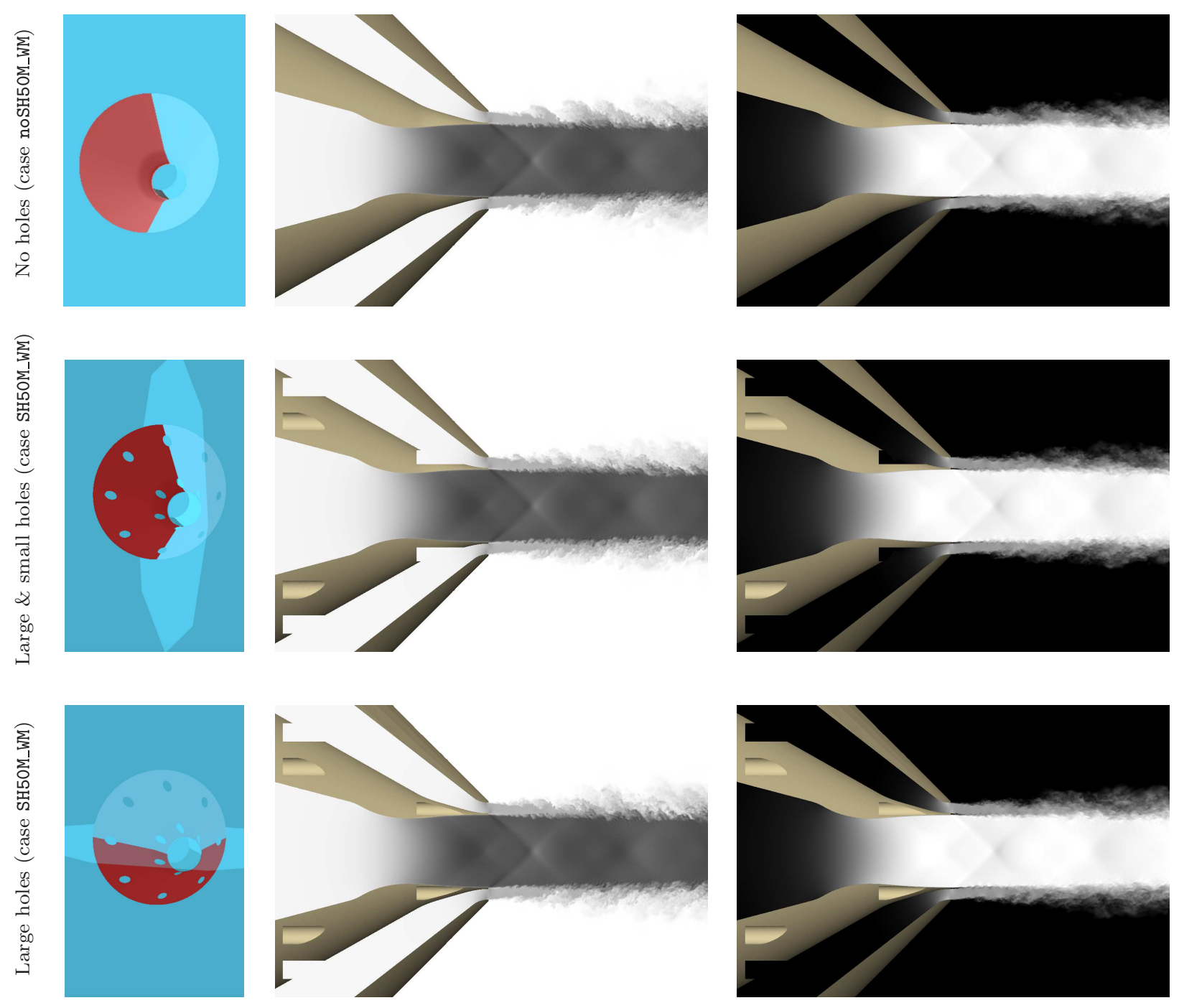

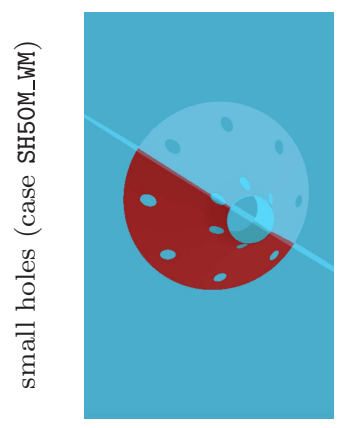

Plane location

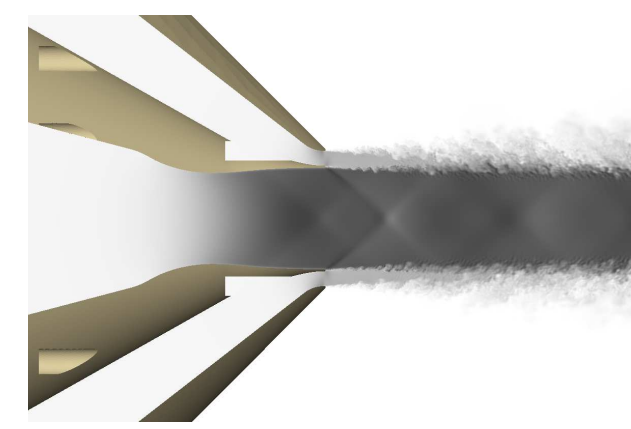

Temperature

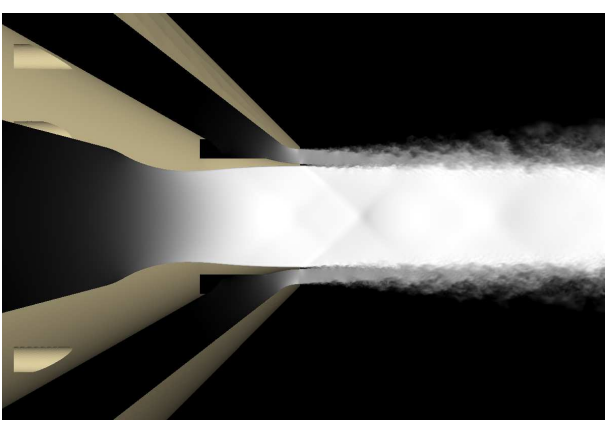

Velocity magnitude

Figure 10. Instantaneous flow field near the nozzle exit in different planar cuts for the LES with and without the nozzle internal screw holes. 

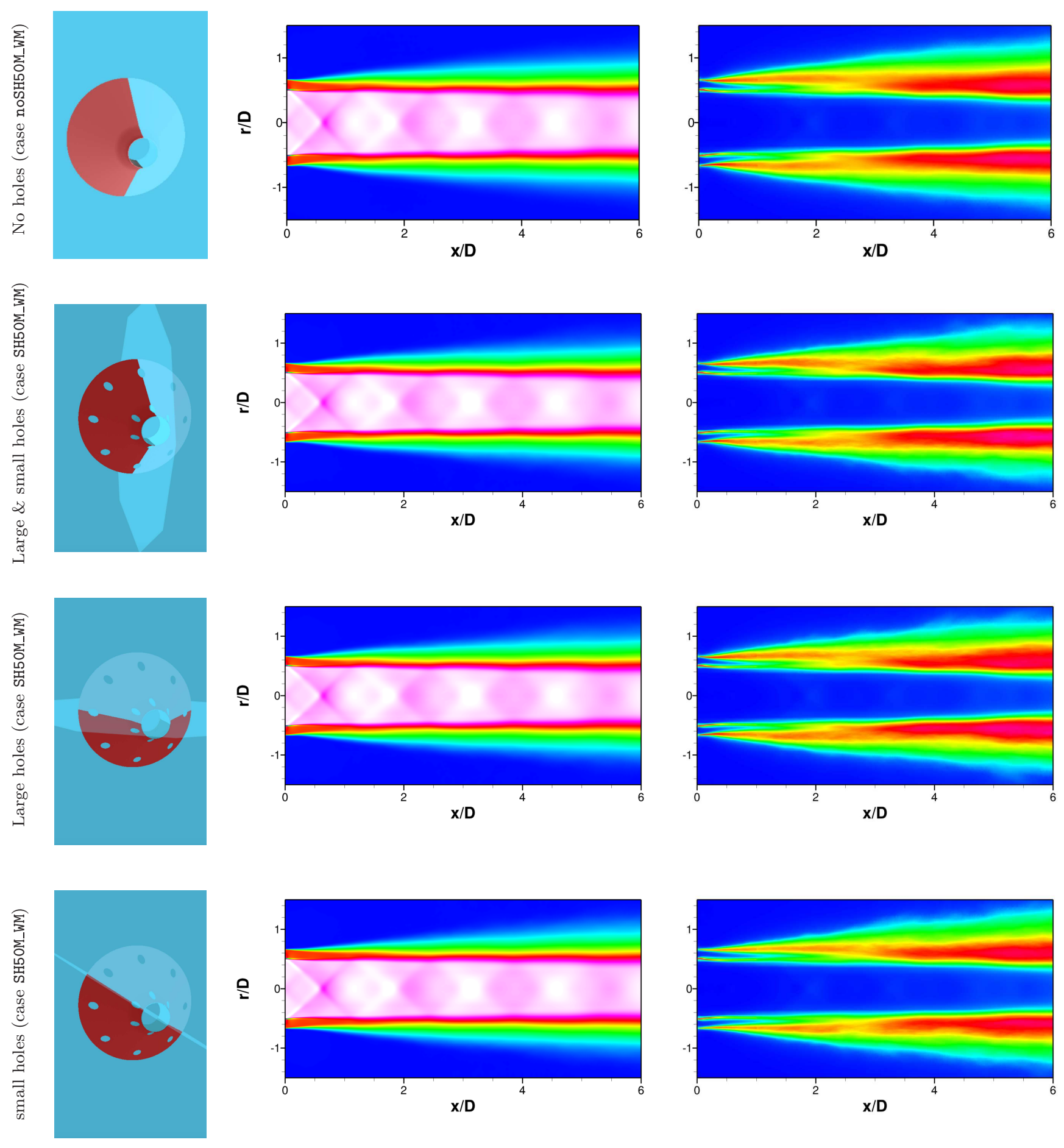

Plane location

Time average

RMS

Figure 11. Zoom-in view near the nozzle exit of the streamwise velocity statistics in different planar cuts for the LES with and without the nozzle internal screw holes (see figure 9 for contour levels). 
improve the statistical convergence of the results. For both cases, the spectra feature a bump around $S t \approx 0.3$ at the upstream angle $\phi=60^{\circ}$ which corresponds to the broadband shock-associated noise (BBSAN) and tends to confirm that the nozzle is operating slightly off design. As predicted by theory and observed in the present simulations, the BBSAN shifts to higher frequency and decreases in amplitude as function of the direction of radiation. ${ }^{19-21}$

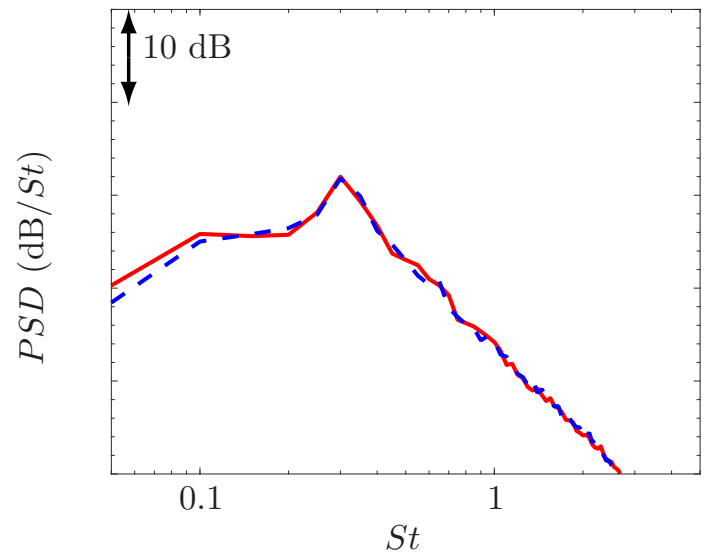

(a) $\phi=60^{\circ}$

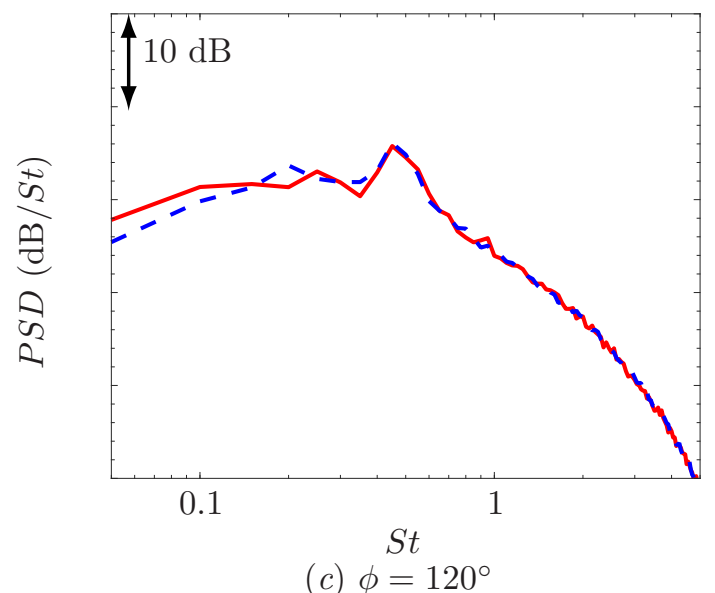

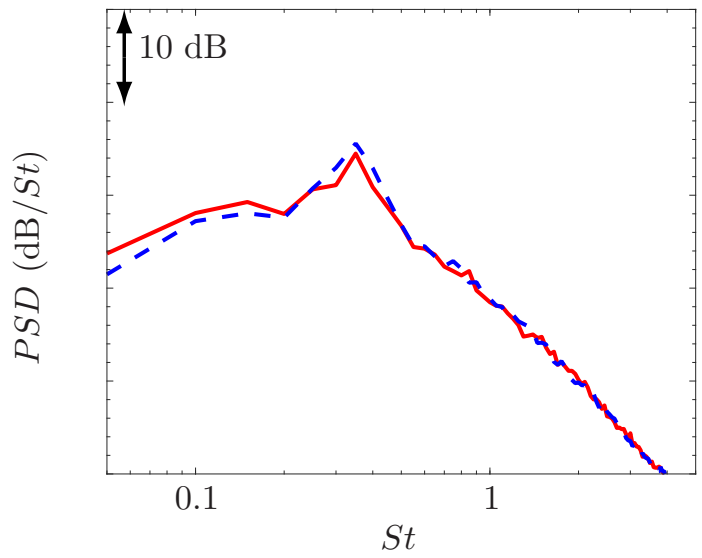

(b) $\phi=90^{\circ}$

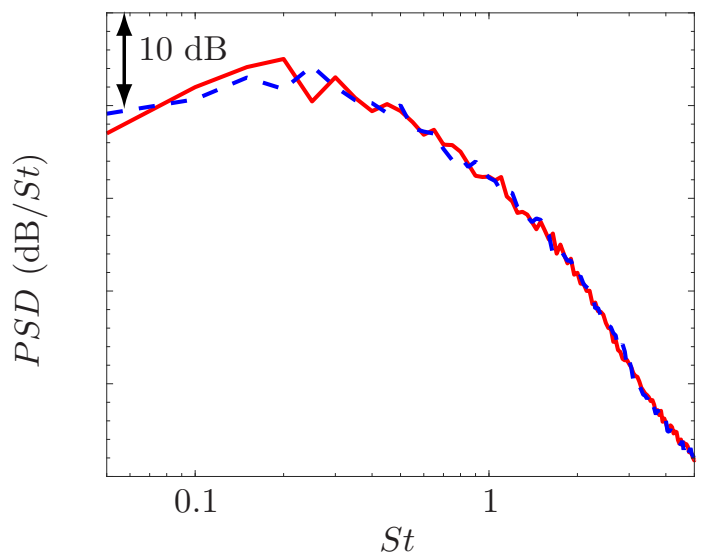

(d) $\phi=150^{\circ}$

Figure 12. Near-field noise predictions for the simulations with ( ---- SH50M_WM) and without ( noSH50M_WM) the internal screw holes.

\section{Conclusions}

The paper presents an overview of the novel mesh generation paradigm based on the computation of Voronoi diagrams recently developed at Cascade Technologies and its application to jet aeroacoustic predictions for complex nozzle. Specifically, large eddy simulation are being performed for a cold dual-stream jet issued from cylindrical co-axial nozzles. The configuration results in an ideally-expanded supersonic jet with Mach number $M_{1}=1.55$ for the primary stream and subsonic jet with $M_{2}=0.9$ for the secondary stream. The Reynolds number based on the primary jet properties is $R e=2.9 \times 10^{6}$. Near-wall refinement and wall modeling are applied on the nozzle interior surfaces to capture the high-Reynolds number wall-bounded flow inside the nozzles.

The geometry and all the operating conditions match experiments conducted at the Georgia Institute of Technology, including the internal screw holes used to fasten the nozzles. While these slanted cylindrical holes over which the secondary stream flows could present a challenge for traditional meshing approaches, such small features are seamlessly captured in the computational mesh and simulation with the Voronoi diagram framework. 
Overall, the preliminary LES results tend to indicate that the internal screw holes have no significant effects on the flow field and radiated noise. As expected, the main characteristics of the present dual-stream configuration with a subsonic annular stream surrounding the primary supersonic stream are a reduced shear-layer growth, a longer potential core and a lack of strong Mach wave radiation. The simulation are being extended to improve the statistical convergence and to complete the comparison with experimental measurements. A long LES database is also being generated for further analysis and modeling of turbulent jets in complex configurations.

\section{Acknowledgments}

The LES studies are supported in part by NAVAIR SBIR projects, under the supervision of Dr. John T. Spyropoulos and by ONR grant N00014-16-1-2445. The main calculations were carried out at DoD HPCMP supercomputer system "Onyx" in ERDC DSRC. The authors would like to thanks Prof. Kris Ahuja, Aharon Karon \& Nick Breen of Georgia Institute of Technology, for providing the nozzle geometry and experimental data for the co-annular jet.

\section{References}

\footnotetext{
${ }^{1}$ Bodony, D. J. and Lele, S. K., "Current Status of Jet Noise Predictions Using Large-Eddy Simulation," AIAA J., Vol. 46, 2008, pp. 346-380.

${ }^{2}$ Lele, S. K. and Nichols, J. W., "A second golden age of aeroacoustics?" Phil. Trans. Royal Soc. A, Vol. 372, No. 2022, 2014.

${ }^{3}$ Slotnick, J., Khodadoust, A., Alonso, J., Darmofal, D., Gropp, W., Lurie, E., and Mavriplis, D., "CFD Vision 2030 Study: A Path to Revolutionary Computational Aerosciences," Tech. Rep. CR 2014-218178, NASA, 2014.

${ }^{4}$ Brès, G. A., Jaunet, V., Le Rallic, M., Jordan, P., Colonius, T., and Lele, S. K., "Large eddy simulation for jet noise: the importance of getting the boundary layer right," AIAA paper 2015-2535, 2015.

${ }^{5}$ Brès, G. A., Jaunet, V., Le Rallic, M., Jordan, P., Towne, A., Schmidt, O. T., Colonius, T., Cavalieri, A. V. G., and Lele, S. K., "Large eddy simulation for jet noise: azimuthal decomposition and intermittency of the radiated sound," AIAA paper 2016-3050, 2016.

${ }^{6}$ Brès, G. A., Ham, F. E., Nichols, J. W., and Lele, S. K., "Unstructured Large Eddy Simulations of Supersonic Jets," AIAA J., Vol. 55, No. 4, 2017, pp. 1164-1184.

${ }^{7}$ Weatherill, N., "Delaunay triangulation in computational fluid dynamics," Computers 85 Mathematics with Applications, Vol. 24, No. 5, 1992, pp. 129-150.

${ }^{8} \mathrm{Du}$, Q., Faber, V., and Gunzburger, M., "Centroidal Voronoi tessellations: applications and algorithms," SIAM review, Vol. 41, No. 4, 1999, pp. 637-676.

${ }^{9}$ Brès, G. A., Jordan, P., Jaunet, V., Le Rallic, M., Cavalieri, A. V. G., Towne, A., Lele, S. K., Colonius, T., and Schmidt, O. T., "Importance of the nozzle-exit boundary-layer state in subsonic turbulent jets," Accepted for publication in Journal of Fluid Mechanics, 2018.

${ }^{10}$ Papamoschou, D., "Mach Wave Elimination in Supersonic Jets," AIAA J., Vol. 35, No. 10, 1997, pp. $1604-1611$.

${ }^{11}$ Freund, J. B., "Proposed Inflow/Outflow Boundary Condition for Direct Computation of Aerodynamic Sound," AIAA J., Vol. 35, No. 4, 1997, pp. 740-742.

${ }^{12}$ Mani, A., "Analysis and optimization of numerical sponge layers as a nonreflective boundary treatment," Journal of Computational Physics, Vol. 231, 2012, pp. 704-7016.

${ }^{13}$ Vreman, A., "An eddy-viscosity subgrid-scale model for turbulent shear flow: Algebraic theory and applications," Physics of Fluids, Vol. 16, Jan 2004, pp. 3570.

${ }^{14}$ Larsson, J., Kawai, S., Bodart, J., and Bermejo-Moreno, I., "Large eddy simulation with modeled wall-stress: recent progress and future directions," JSME Mech. Eng. Reviews, Vol. 3, 2016.

${ }^{15}$ Bose, S. T., . P. G. I., "Wall-Modeled Large-Eddy Simulation for Complex Turbulent Flows," Annual Review of Fluid Mechanics, Vol. 50, 2018, pp. 535-561.

${ }^{16}$ Lockard, D. P., "An efficient, two-dimensional implementation of the Ffowcs Williams and Hawkings equation," J. Sound Vib., Vol. 229, 2000, pp. 897-911.

${ }^{17}$ Ffowcs Williams, J. E. and Hawkings, D. L., "Sound generation by turbulence and surfaces in arbitrary motion," Philosophical Transactions of the Royal Society of London. Series A, Mathematical and Physical Sciences, Vol. 264, 1969, pp. 321342.

${ }^{18}$ Shur, M. L., Spalart, P. R., and Strelets, M. K., "Noise prediction for increasingly complex jets. Part I: Methods and tests," Int. J. Aeroacoust., Vol. 4, No. 3-4, 2005, pp. 213-246.

${ }^{19}$ Harper-Bourne, M. and Fisher, M. J., "The noise from shock waves in supersonic jets," Tech. Rep. CP 131, AGARD, 1974.

${ }^{20}$ Tam, C. K. W. and Tanna, H. K., "Shock associated noise of supersonic jets from convergent-divergent nozzles," J. Sound Vib., Vol. 81, 1982, pp. 337-358.

${ }^{21}$ Tam, C. K. W., Seiner, J. M., and Yu, J. C., "Proposed Relationship Between Broadband Shock Associated Noise and Screech Tones," J. Sound Vib., Vol. 110(2), 1986, pp. 309-321.
} 